\title{
Christian Koller Leiden und «Lust» in der Fremdenlegion
}

\begin{abstract}
Der britische Ex-Fremdenlegionär Anthony Delmayne schilderte in seinen Memoiren folgende Episode, die sich in der Zwischenkriegszeit im Zusammenhang mit der Bekämpfung von Rebellen im französischen Mandatsgebiet Syrien zugetragen hatte:
\end{abstract}

[...] wir rannten in das Dorf, um die Leute einzukreisen. Ich bog um eine Ecke, und eine Frau, die in einer Tür stand, flehte mich an, sie nicht zu töten und stattdessen ihre Tochter flachzulegen. Das Mädchen war etwa fünfzehn und hübsch, und ich hatte seit längerer Zeit nicht mehr. Ich durchsuchte das Haus - es konnte ja eine Falle sein, und das Mädchen fügte sich mir, während die Mutter an der Türe Wache hielt. Ich behielt meinen Säbel neben mir, der immer noch blutverschmiert war, vielleicht vom Vater oder den Brüdern des Mädchens, und schaute immer wieder über meine Schulter, ob sich jemand anschleichen und mich von hinten erdolchen wollte. Als ich fertig war, erinnerte ich mich meiner Pflicht, befahl dem Mädchen aufzustehen und führte sie und ihre Mutter zum Versammlungsort, wo die anderen Dorfbewohner umzingelt waren. ${ }^{1}$

Anthony Delmayne: Sahara Desert Escape, London 1958, 17: «[...] we [...] ran into the village to round up the people. I turned a corner, and a woman in a doorway begged me not to kill her but to zigzig her daughter instead. The girl was about fifteen and comely, and it had been a long time. I searched the house in case it was a trap and the girl submitted to me while her mother kept watch at the door. I kept my sabre beside me, still smeared with the blood perhaps of the girl's father or brothers, and every now and then I looked over my shoulder in case someone should creep up and stab me in the back. When I had finished I remembered my duty, ordered the girl to get up, and took her and her mother to the assembly point where the other villagers had been rounded up.» (eigene Übersetzung). 
Diese Geschichte einer Vergewaltigung bei einem Counterinsurgency-Einsatz ist exemplarisch für viele Aktionen der Fremdenlegion im Zeitalter des Kolonialismus und führt direkt zum Thema Lust und Leiden. Die Fremdenlegion als Instrument imperialer Herrschaft fügte unzähligen Menschen in Asien und Afrika Leid $\mathrm{zu}$; zugleich litten viele Fremdenlegionäre aber auch unter ihrer Situation, die unter anderem ständige Lebensgefahr und längere Phasen sexueller Abstinenz beinhaltete. Die Lust des Legionärs führte dabei zuweilen zu sexueller und anderer Gewalt, zuweilen wurde sie durch Praktiken befriedigt, die im Widerspruch zu den zeitgenössischen Moralvorstellungen standen. Es sind diese drei Punkte - der leidende Legionär, der Leid zufügende Legionär und die «Lust» des Legionärs -, die ich im Folgenden nach einem einführenden Abriss der Geschichte der Fremdenlegion diskutieren möchte. Ich stütze mich dabei sowohl auf Selbstzeugnisse (Briefe, Interviews, autobiographische Aufzeichnungen) der Legionäre als auch auf die Ergebnisse der einschlägigen militärhistorischen und kulturwissenschaftlichen Forschung.

\section{Geschichte der Fremdenlegion im Überblick}

Die Fremdenlegion ist ein, wenn auch vielleicht illegitimes, Kind des europäischen Revolutionsjahres 1830. ${ }^{2}$ In Frankreich stürzte die Julirevolution das Restaurationsregime und ersetzte es durch eine etwas liberalere Monarchie unter dem «Bürgerkönig» Louis-Philippe I. Aber nicht nur im Hexagon kam es zu einem Umsturz, auch in zahlreichen anderen Regionen Europas regten sich liberale und nationalistische Kräfte. Hinter der Gründung einer aus Ausländern gebildeten militärischen Einheit durch die neue

2 Das Folgende nach Douglas Porch: The French foreign legion. A complete history, New York, London 1991; Eckard Michels: Deutsche in der Fremdenlegion. Mythen und Realitäten 1870-1965, Paderborn 1999; Christian Koller: Die Fremdenlegion. Kolonialismus, Söldnertum, Gewalt, 1831-1962, Paderborn 2013. 
französische Regierung am 10. März 1831 stand kein längerfristiges sicherheitspolitisches oder gar geostrategisches Kalkül. Vielmehr waren kurzfristige Erwägungen ausschlaggebend: Aufgrund der europaweiten Unruhen befanden sich 1831 zahlreiche politische Flüchtlinge in Frankreich, die von der Regierung als potentieller Unruheherd wahrgenommen wurden. Die Schaffung einer Söldnertruppe für den überseeischen Einsatz bot die Gelegenheit, diese Personen ausser Landes zu schaffen, ohne Frankreichs Ansehen eines liberalen Asyllandes bei den fortschrittlichen Kräften Europas zu gefährden. Eine zweite Rekrutierungsbasis stellten die zahlreichen arbeitslos gewordenen Söldner dar, die in den königlichen Schweizerregimentern und im Regiment Hohenlohe bis zu deren Auflösung im Gefolge der Julirevolution gedient hatten. Im Unterschied zu ihren Vorläufern durfte die 1831 gegründete Fremdenlegion nicht im Inland eingesetzt werden und erschien damit entsprechend den liberalen Forderungen nicht als ein Machtinstrument der Krone, sondern der gesamten Nation, obgleich ihre Zusammensetzung der nationalen Konzeption der einer Armee von Wehrpflichtigen diametral entgegenstand.

Die Fremdenlegion war von Beginn an primär ein Instrument des Imperialkrieges. Als erstes gelangte sie in den 1830er und 1840er Jahren bei der Eroberung Algeriens zum Einsatz. Algerien sollte denn auch bis zur Unabhängigkeit des Landes im Jahr 1962 ihre «Heimat» sein, in der ihr Hauptquartier stand und wo die Rekruten ihre Grundausbildung durchliefen. Der nächste Einsatz der Fremdenlegion nach der (beziehungsweise parallel zur) Eroberung Algeriens fand dann allerdings in Europa statt. 1835 überliess Frankreich die Legion per Vertrag der spanischen Regierung unter Isabella II. Im Thronfolgekrieg (1834-1839), den die Königin, unterstützt durch Grossbritannien und Frankreich, gegen die Karlisten führte, kam die erste Generation der Fremdenlegionäre fast vollumfänglich ums Leben. Nur 250 von ursprünglich 6000 Mann kehrten 1839 nach Frankreich zurück.

In den 1850er Jahren wurde die Fremdenlegion zum ersten Mal in Konflikte innerhalb des europäischen Mächtekonzerts involviert. 
100 Christian Koller: Leiden und «Lust» in der Fremdenlegion

Von 1854 bis 1856 kämpfte sie im Krimkrieg gegen Russland und etablierte sich definitiv als Elitetruppe für Einsätze sowohl in kolonialen als auch europäischen Kriegen. 1859 nahm sie am Zweiten Italienischen Unabhängigkeitskrieg gegen Österreich teil. Von 1863 bis 1867 beteiligte sie sich an der französischen Mexikoexpedition, dem vergeblichen Versuch Napoleons III., Erzherzog Ferdinand Maximilian Joseph von Österreich als Kaiser von Mexiko zu installieren. Im Deutsch-Französischen Krieg (1870/71) gelangte sie erstmals zur Verteidigung des französischen Mutterlandes zum Einsatz. Entgegen dem Verbot des Einsatzes im Innern wurden im Mai 1871 auch Legionseinheiten bei der blutigen Niederschlagung des Pariser Commune-Aufstandes herangezogen.

In der Folgezeit spielte die Legion eine wesentliche Rolle beim Ausbau und bei der Konsolidierung französischer Kolonialherrschaft in Afrika und Südasien, so von 1883 bis 1897 bei der vollständigen Eroberung Indochinas, 1892 bei der Unterwerfung von Dahomey und 1895 bei der Eroberung von Madagaskar. Bei letzterer Operation gab General Jacques Charles Duchesne für die Legionäre das seither sprichwörtliche Motto «marschiere oder krepiere» aus. Ab 1907 war die Fremdenlegion im zunehmend in die französische Einflusssphäre eingegliederten, aber auch vom Deutschen Reich beanspruchten Marokko präsent.

Im Ersten Weltkrieg kam die Söldnertruppe erneut bei der Verteidigung des Mutterlandes zum Einsatz. Ausländer, die für Frankreich kämpfen wollen, wurden als engagés volontaires pour la durée de la guerre in die Fremdenlegion integriert, so dass sich deren Bestand bis Mitte 1915 auf 22000 Mann beinahe verdoppelte. In der Zwischenkriegszeit setzte sich der Gebrauch der Fremdenlegion als Machtinstrument des französischen Kolonialimperialismus fort. Wichtigstes Einsatzgebiet war Marokko, wo die Legion bei der endgültigen Unterwerfung aller Landesteile und dann 1925/26 zusammen mit spanischen Einheiten im Krieg gegen Mohammed Abd El-Krims aufständische Rif-Republik zum Einsatz gelangte. Daneben gab es etwa auch Operationen in Indochina und dem auf der Pariser Friedenskonferenz erworbenen französischen 
Mandatsgebiet Syrien. Beim Ausbruch des Zweiten Weltkrieges nahm Frankreich erneut Ausländer für die Dauer des Krieges in die Legion auf. 1939/40 meldeten sich Zehntausende von Freiwilligen aus über 50 Ländern. Am Vorabend des deutschen Angriffs auf Frankreich erreichte der Mannschaftsbestand der Fremdenlegion mit 49000 Mann seinen historischen Höchststand.

Von 1945 bis in die 1960er Jahre diente die Fremdenlegion als militärisches Instrument in dem vergeblichen Versuch, das durch den Zweiten Weltkrieg erschütterte französische Kolonialimperium zusammenzuhalten. Legionäre gelangten in den Dekolonisationskämpfen in Indochina (1946-1954), Madagaskar (1945-1948), Tunesien (1952-1955), Marokko (1953-1955) und Algerien (Massaker von 1945, Algerienkrieg 1954-1962) sowie im Suezkrieg (1956) zum Einsatz. Die wichtigsten Einsätze dieser Epoche waren diejenigen im Indochina- und im Algerienkrieg. Von den 488500 Soldaten, die im Indochinakrieg auf französischer Seite kämpften, waren 15 Prozent Fremdenlegionäre. Ein bedeutender Teil der restlichen französischen Truppen bestand aus Afrikanern und Vietnamesen. Bei der entscheidenden Schlacht von Điện Biên Phủ im Frühjahr 1954 stellte die Fremdenlegion den Kern der französischen Truppen. Der Algerienkrieg und die algerische Unabhängigkeit im Jahr 1962 stürzten die Fremdenlegion, die ihre «Heimat» seit 130 Jahren in Algerien gehabt hatte, in eine existentielle Krise. Am Generalsputsch vom 21. April 1961 in Algier, der die angelaufenen Verhandlungen von de Gaulles Regierung mit dem algerischen Front de Libération Nationale torpedieren sollte, war das $1^{\text {er }}$ Régiment Étranger de Parachutistes prominent beteiligt. Nach dem Zusammenbruch des Putsches wurde dieses Regiment unverzüglich aufgelöst, und auch der Fortbestand der Fremdenlegion insgesamt stand zunächst in Frage.

Schon bald offenbarte sich, dass die Grande Nation auch nach dem Ende ihres Kolonialreiches eine globalpolitische Rolle zu spielen gedachte. Die Fremdenlegion hatte in dieser Konstellation weiterhin Platz in den militärischen Planungen, musste sich allerdings adaptieren und mutierte zu einer radikal verkleinerten Eliteeinheit 
mit stark erhöhten Eintrittsanforderungen. Ihre Einheiten wurden neu in Südfrankreich, auf Korsika, in Djibouti, auf Madagaskar, auf Tahiti und in Französisch-Guyana stationiert. Neben traditionellen Kriegseinsätzen (zum Beispiel im Golfkrieg von 1991) traten out-of-area-Operationen (wie 1978 die Befreiung von 1200 europäischen Geiseln aus der Hand von Rebellen im zairischen Kolwezi), wiederholte Interventionen in Afrika, der Objektschutz am Weltraumbahnhof in Französisch-Guayana und Einsätze im Rahmen von UNO- oder NATO-Missionen (beispielsweise im Libanon, Bosnien, Kosovo oder Afghanistan), von humanitärer Hilfe (Ruanda 1994) und zur Katastrophenhilfe (zum Beispiel nach dem Tsunami in Südostasien 2004).

Die Grösse der Fremdenlegion änderte sich in ihrer Geschichte vielfach und oszillierte zwischen 3000 und 49000 Mann. Auch die Zusammensetzung war nicht konstant, insbesondere was die Nationalität der zu Rekrutierenden betraf. ${ }^{3}$ Das Angebot an Eintrittswilligen wurde sowohl quantitativ als auch bezüglich ihrer Herkunftsgebiete von einer Vielzahl politischer, wirtschaftlicher, sozialer und kultureller Faktoren beeinflusst, während auf der Nachfrageseite sich wandelnde Parameter wie imperiale Ambitionen, die politische Grosswetterlage Europas, ausländer- und flüchtlingspolitische Erwägungen und die Sorge um Effizienz und Zuverlässigkeit der Fremdenlegion die Rekrutierungspolitik bestimmten. Das Grundstatut der Fremdenlegion von 1831 schuf verschiedene Grundlagen, nach denen die Einheit in den folgenden Jahrzehnten funktionieren und ihre Soldaten rekrutieren sollte, während sich andere institutionelle Rahmenbedingungen erst im Laufe der Zeit herausbildeten. 1864 wurde die Dienstzeit auf fünf Jahre mit Verlängerungsmöglichkeit festgesetzt. Eine Besonderheit war das sogenannte Anonymat, die Möglichkeit, beim Eintritt eine falsche Identität anzugeben. Im 19. Jahrhundert entwickelte sich zunächst

$3 \quad$ Vgl. Christian Koller: Recruitment Policies and Recruitment Experiences of the French Foreign Legion, in: Nir Arielli, Bruce Collins (eds.): Transnational Soldiers. Foreign Military Enlistment in the Modern Era, Basingstoke 2013, 87-104. 
103 Christian Koller: Leiden und «Lust» in der Fremdenlegion

das Gewohnheitsrecht, dass Eintrittswillige, die keine Papiere vorlegen konnten oder wollten, trotzdem aufgenommen werden konnten. 1911 wurde das Anonymat dann explizit kodifiziert.

Das Anonymat verschaffte der Fremdenlegion rasch den Ruf, ein Sammelbecken von Kriminellen und Abenteurern zu sein. Wer waren aber die Legionsfreiwilligen? Der reformierte Schweizer Seelsorger Eduard Blocher, der in den 1890er Jahren mehrere Jahre bei der Garnison des ersten Fremdenregiments in Sidi-Bel-Abbès gewirkt hatte, meinte, in erster Linie fänden sich unter den Legionären «sehr viele Arbeitslose oder auch einfach Neugierige, von echt- und urgermanischer Wanderlust über die Grenze getriebene, zum Teil recht unerfahrene Bürschlein». ${ }^{4}$ Effektiv stammte die überwiegende Mehrheit der rund 500000 Männer, die bis 1962 in die Fremdenlegion eintraten, aus den europäischen Unterschichten, und ein Grossteil von ihnen unterzeichnete aus materiellen Gründen, Armut, Arbeits- und Perspektivlosigkeit einen Fünfjahreskontrakt zugunsten des französischen Staates. Zwar waren viele von ihnen in der Tat schon mit dem Gesetz in Konflikt gekommen, in aller Regel aber wegen Bagatelldelikten. ${ }^{5}$

Ein zweites Rekrutierungsreservoir waren politische Flüchtlinge. ${ }^{6}$ Nach den europäischen Revolutionswellen von 1830 und 1848 traten zahlreiche polnische und deutsche Flüchtlinge in die Legion ein. 1850 versuchte der Bundesrat, in der Schweiz anwesende 1848er-Flüchtlinge in die Fremdenlegion abzuschieben. ${ }^{7}$ Weitere

Eduard Blocher: Von der französischen Fremdenlegion, in: Wissen und Leben 13 (1913/14) 327-344, hier 337-339.

5 Christian Koller, Peter Huber: Armut, Arbeit, Abenteuer - Sozialprofil und Motivationsstruktur von Schweizer Söldnern in der Moderne, in: Vierteljahrschrift für Sozial- und Wirtschaftsgeschichte 102/1 (2015) 3051; Peter Huber: Fluchtpunkt Fremdenlegion. Schweizer im Indochinaund im Algerienkrieg, 1945-1962, Zürich 2017.

$6 \quad$ Vgl. Christian Koller: «Gerade vom Professorenstuhl heruntergestiegen und in die Uniform hineingeschlüpft». Söldnertruppen als Asylort im 19. und frühen 20. Jahrhundert, in: Portal Militärgeschichte http:/ / portal-militaergeschichte.de/ koller soeldnertruppen (11.9.2018)

7 Vgl. Kreisschreiben des Schweizerischen Bundesrates an die Regierungen sämmtlicher Stände, betreffend 1) die Aufnahme der Flüchtlinge in die 
Flüchtlingswellen erreichten die Legion nach dem Ende des Ersten Weltkriegs und des Russischen Bürgerkriegs und dann in den 1930er Jahren infolge der zunehmenden Repression in NaziDeutschland sowie nach dem Ende des Spanischen Bürgerkriegs. Aussteiger mit bürgerlichem oder gar aristokratischem Hintergrund waren dagegen in der Fremdenlegion insgesamt Ausnahmen. Allerdings gab es ein paar spektakuläre Fälle. So stellte sich der 1897 eingetretene und 1898 an Typhus verstorbene Legionär Albrecht Nordmann nach seinem Tod als Prinz Albert Friedrich von Hohenzollern, Cousin Kaiser Wilhelms II., heraus.

\section{Der leidende Legionär}

Der Eintritt in die Legion stellte bei vielen einen Versuch dar, ihrem materiellen oder seelischen Leiden zu entkommen. Ein drastisches Beispiel war der arbeitslose und verschuldete Schweizer Gärtner Bernhard Pfyl, der 1947 kurz nach seinem Legionseintritt in einem Brief an Bundespräsident Philipp Etter schrieb: «Ich bin moralisch so tief gesunken, so dass ich zum Entschluss kam, mich in die Legion zu melden, damit mein Leben gekürzt wird.» ${ }^{8}$ Die militärische Grundausbildung stellte in der Regel jedoch alles andere als das Ende des Leidens dar. Der 1950 in die Legion eingetretene Brite Henry Ainley erwähnte in seinen Memoiren, dass im ersten Drittel der neunwöchigen Rekrutenschule

die meisten von uns zu pochenden Klumpen aus Schmerz und von Erschöpfung geplagtem Fleisch reduziert wurden, die sich nach nichts anderem als nach Schlaf sehnten. Wir hatten keine Zeit zu trinken, wenig Zeit zu rauchen und überhaupt keine, um uns selbst zu bemitleiden. Nachdem unsere Instruktoren uns in einen entsprechenden Zustand der Empfänglichkeit versetzt hatten, fuhren sie fort, uns die

Fremdenlegion in Algerien, 2) die dermalige Anzahl der Flüchtlinge in der Schweiz, in: Bundesblatt 1850, 96-99; Journal de Genève, 4.3.1850 u. 15.3.1850.

8 Schweizerisches Bundesarchiv (Bern) E 5330-1/1975/95/1948/9 Militärgericht: Pfyl, Bernhard, 1948. 
105 Christian Koller: Leiden und «Lust» in der Fremdenlegion

feineren Künste des Rechtsum kehrt! und Linksum kehrt!, des Salutierens, des Marschierens im Gleichschritt und des Waffendrills einzuimpfen. ${ }^{9}$

Die meisten Legionäre litten nach etwa einem halben Jahr an Depressionen, die im Legionsjargon als cafard bezeichnet wurden. Verursacht wurden diese depressiven Anfälle von Resten der Vorlegionsbiographie, die durch die Grundausbildung nicht ganz zerstört wurden, gepaart mit legionsspezifischen Fährnissen wie der strengen Disziplin, harten körperlichen Anforderungen, ungewohntem Klima und der oft unerträglichen Langeweile in abgelegenen Garnisonsposten, die Friedrich Glauser in seinem autobiographischen Roman Gourrama eindrücklich beschrieben hat. ${ }^{10}$ Der cafard (wörtlich «Kakerlake», «Schabe») befiel fast jeden Legionär mindestens einmal und konnte zu Kurzschlusshandlungen führen, doch verschwand er in der Regel ohne psychiatrische Behandlung wieder. In der Legionshierarchie war denn auch teilweise schlicht vom «faire sa maladie» des Rekruten die Rede. ${ }^{11}$ Eine gewisse Ähnlichkeit scheint der cafard mit einem als nostalgia oder delirium melancholicum bezeichneten Syndrom frühneuzeitlicher Söldner zu haben, das vom 17. bis 19. Jahrhundert Gegenstand umfangreicher medizinischer und literarischer Diskurse war. ${ }^{12}$ Die Erkenntnisse neuerer Forschungen zur Militärpsychiatrie bezüglich der Situativität von Syndromen, die in jedem Krieg ein

$9 \quad$ Henry Ainley: In order to die. With the French Foreign Legion in Indochina, London 1955, 16: «the majority of us were reduced to throbbing lumps of pain and fatigue-racked flesh, whose only idea was sleep. We had no time to drink, little to smoke in, and none to feel sorry for ourselves. Having reduced us to an appropriate state of receptivity, our instructors then proceeded to instil in us the finer arts of doing an aboutturn, saluting, marching in step and rifle drill.» (eigene Übersetzung).

10 Friedrich Glauser: Gourrama. Ein Roman aus der Fremdenlegion, hg. und mit einem Nachwort von Bernhard Echte, Zürich 1997.

11 Vgl. z.B. Service Historique de la Défense (Vincennes) 10H 375 Rapport

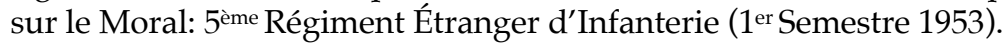

12 Vgl. Christian Schmid-Cadalbert: Heimweh oder Heimmacht. Zur Geschichte einer einst tödlichen Schweizer Krankheit, in: Schweizerisches Archiv für Volkskunde 89 (1993) 69-85. 
106 Christian Koller: Leiden und «Lust» in der Fremdenlegion

spezifisches Repertoire an Erklärungskonzepten hervorgebracht haben, ${ }^{13}$ legen indessen nahe, den cafard als ein spezifisches Syndrom der Fremdenlegion mit ihrer weitgehend einmaligen Kombination aus einer heterogenen Zusammensetzung, überseeischen Einsatzorten und einer Dominanz sozial und kulturell deprivierter und / oder psychisch labiler Soldaten zu identifizieren.

Verschiedene Tagebuch-, Brief- und Memoirenschreiber haben sich über den cafard Gedanken gemacht und Ursachen, Symptome und Gegenmittel erörtert. Der Schweizer Willy Zurflüh beschrieb in einem Brief das Syndrom als «[...] Heimweh [...], moralische Momente wo [sic!] fürchterlich sind [...]. Ich habe manchmal Stunden weise geweint, nichts konnte es aufhalten [...].» ${ }^{14}$ Gemäss dem Briten Colin John war der cafard

ein Zustand mentaler Depression, in dem alle Sünden und Tragödien des vergangenen Lebens wie Berge aufragen und die Zukunft rabenschwarz erscheint. Fast alle Legionäre leiden am cafard, besonders während der ersten sechs Monate oder des ersten Jahres ihres Dienstes. Viele betrinken sich massiv, um ihre depressiven Gedanken loszuwerden. Andere ziehen sich nur in sich zurück und arbeiten an ihren Problemen, so gut das möglich ist, in einem versteinerten und undurchdringbaren Schweigen. ${ }^{15}$

Sein Landsmann Simon Murray charakterisierte den Zustand als die Empfindung von Millionen kleiner Käfer, die einem im Kopf herumkriechen, und dieses Gefühl muss unter Kontrolle gehalten werden,

13 Vgl. Edgar Jones, Simon Wessely: War Syndromes. The impact of culture on medically unexplained Symptoms, in: Medical History 49 (2005) 55-78. Schweizerisches Bundesarchiv (Bern) E 5330-1/1975/95/1951/1075 Militärgericht: Ammann, Heinz, 1951.

15 Colin John: Nothing to Lose, London 1956, 144: «a state of mental depression in which all the sins and tragedies of your past life loom up like mountains before you and the future looks very black indeed. Nearly all legionnaires suffer from le cafard, particularly during their first six months or year of service. Many of them drink heavily in order to get away from their depressive thoughts. Others merely retire into themselves and work out their problems, to the extent that it is possible to work them out, in a stony and impenetrable silence» (eigene Übersetzung). 
107 Christian Koller: Leiden und «Lust» in der Fremdenlegion

denn es ist ein Signal, dass man sich in einem Zustand befindet, der dem Wahnsinn unmittelbar vorangeht. Le cafard ist ein Parasit, der sich aus dem Gemüt ernährt, den Geist zersetzt und den Menschen schliesslich zu einem Wrack macht. ${ }^{16}$

Neben diesem seelischen war für den Legionär auch das körperliche Leiden allgegenwärtig. Die physische Härte des Dienstes unter schwierigen klimatischen Bedingungen wurde noch gesteigert durch eine strikte Disziplin, die bis weit ins 20. Jahrhundert hinein auch Körperstrafen einschloss. Die im 19. Jahrhundert berüchtigtste und in zahlreichen Selbstzeugnissen erwähnte ${ }^{17}$ Körperstrafe in der Legion war das tagelange Zusammenbinden von Händen und Füssen auf dem Rücken, die so genannte crapaudine (von Französisch crapaud, Kröte). Diese Strafe wurde im späten 19. Jahrhundert eingeschränkt und Anfang des 20. Jahrhunderts ganz verboten, ${ }^{18}$ sie blieb aber noch jahrelang im kommunikativen Gedächtnis der Fremdenlegionäre haften. ${ }^{19}$ Wie häufig sie nach dem Verbot noch

Simon Murray: Legionnaire. An Englishman in the French Foreign Legion, London 1978, 51-52: «the sensation of millions of tiny beetles crawling around inside one's head and it is a feeling that must be held in check because it is a signal that one is in a condition that immediately precedes madness. Le cafard is a parasite that feeds on the mind, corrodes the spirit and ultimately reduces the physical being to a wreck» (eigene Übersetzung).

17 Zum Beispiel Wilhelm Bolliger: Aus dem Leben und Treiben der Fremdenlegion in Afrika und Tonkin, 1884-1886. Nach eigenen Erlebnissen wahrheitsgetreu geschildert, Basel 1889, 8; Hans Lüthi: Erinnerungen an eine fünfjährige Dienstzeit in der französischen Fremdenlegion mit besonderer Berücksichtigung der daselbst bestehenden allgemeinen $\mathrm{Zu}$ stände (Algier, Tonkin 1880-1885), Bern [1887], 9-10; Raimund Anton Premschitz: Meine Erlebnisse als Fremdenlegionär in Algerien, Metz 1904, 121-122; Frederic Martyn: Life in the Legion. From a Soldier's Point of View, London [1911], 36 u. 215-216.

F. Martyn: Life in the Legion, 215; E. Michels: Deutsche in der Fremdenlegion, 58. Gemäss Laffin wurde diese Strafmethode erst 1920 verboten und war auch danach noch in den Strafbataillonen der Legion in Gebrauch (John Laffin: The French Foreign Legion, London 1974, 27). meinen Tagebuchblättern, Zürich 1921, 59. 
illegal angewandt wurde, ist unklar. Jahrzehnte länger in Gebrauch und auch in kulturellen Verarbeitungen des Legionsthemas bis hin $\mathrm{zu}$ Parodien präsent ${ }^{20}$ war die pelote, das stundenlange Marschieren, Rennen und Kriechen, das mit Rucksäcken, die mit Sand oder Steinen gefüllt waren, häufig in der Hitze zu absolvieren war. Diese Strafe gelangte einerseits bei individuellen Vergehen zur Anwendung, etwa als erste Stufe der Bestrafung von eingebrachten Deserteuren, aber auch bei relativ geringfügigen Verfehlungen gegen die Disziplin. ${ }^{21}$ Andererseits wurde sie auch als Kollektivstrafe eingesetzt.

Auch die Prügelstrafe war noch nach dem Zweiten Weltkrieg in Gebrauch. Der Deutsche Ex-Legionär Peter K. sagte dazu in einem Interview:

Kaum lag man, übergoss einen der Sergeant mit kaltem Wasser und es setzte Prügel - echte Prügel. Nicht eben eine links und eine rechts, sondern draufgedroschen, und anschliessend wieder in die Sonne, wieder exerzieren. [...] Diese Strafen besassen ja noch den Anschein der Legalität, schliesslich hatte man ja - vermeintlich - irgend etwas verbockt. Anders verhielt es sich bei den Züchtigungen durch Offiziere und Offiziersanwärter, die ja alle diese schönen, kleinen Ledergerten trugen. Diese Herren nahmen sich das Recht heraus, wahllos und ohne Grund dem einfachen Legionär mal rechts und links einen überzuziehen. Bei wem wollte man sich beschweren? - Prügel gehörten zur Tagesordnung. ${ }^{22}$

Auch nach der Transformation der Fremdenlegion in den 1960er Jahren verschwanden die harschen Disziplinierungsmethoden nicht vollständig. 1977 enthüllte das aufsehenerregende Buch L'Épreuve des Journalisten Henry Allainmat, dass im Straflager für wegen schwerer Disziplinverstösse verurteilte Legionäre auf Korsika Kör-

Vgl. z.B. René Goscinny, Albert Uderzo: Astérix légionnaire, Neuillysur-Seine 1967, 27-29.

Vgl. z.B. Paul Strupler: Fünf Jahre Fremdenlegion, Frauenfeld, Leipzig 1928, 49; S. Murray: Legionnaire, 57-58, 66 u. 152; Mythos Fremdenlegion Deutsche in Frankreichs Diensten (ZDF 2009), min. 9:12ff.

Peter K.: Wir hatten Gewalt empfangen und gaben sie wieder zurück. Peter K., Legionär von 1946 bis 1950, in: Detlev Michelers: Le boudin. Deutsche Fremdenlegionäre der Nachkriegszeit, Berlin 1990, 19-28, hier 23-25. 
perstrafen und andere psychische und physische Erniedrigungen immer noch an der Tagesordnung waren. ${ }^{23}$ Das deutsche Wochenmagazin Der Spiegel charakterisierte diese Methoden in einer Rezension als «Folter» und «barbarisch». ${ }^{24}$

Schliesslich konnten die Kampfhandlungen das Leiden von Legionären ins Unermessliche steigern. In zahlreichen Selbstzeugnissen tauchen von der Gegenseite begangene Gräueltaten aller Art auf, so das $\mathrm{Zu}$-Tode-Foltern gefangener Legionäre, ${ }^{25}$ das Abschneiden von Nasen, Ohren, Köpfen und Genitalien, ${ }^{26}$ das Ausstechen von Augen ${ }^{27}$ oder die gleichgeschlechtliche Vergewaltigung. ${ }^{28}$ Die detaillierte Beschreibung dieser angeblichen oder tatsächlich erlebten Gräueltaten, die als Topoi im Kolonialdiskurs auch in anderen Kontexten auftauchten, entlastete die Ex-Legionäre bezüglich der von der Fremdenlegion begangenen Grausamkeiten, die dadurch als Vergeltungsmassnahmen entschuldigt werden konnten. Dies führt zum nächsten Thema, dem Legionär als Leiden zufügendem Akteur im Imperialkrieg.

Henry Allainmat: L'Épreuve. Le Bagne de la Légion, Paris 1977. Der Spiegel, 28.3.1977.

F. Martyn: Life in the Legion, 199; H. Seiler: Fünf Jahre in der französischen Fremdenlegion, 48 u. 58; W. Bolliger: Aus dem Leben und Treiben der Fremdenlegion, 33-34; Robert Kaufmann: Feldzug in Afrika, Tonkin \& China nach eigenen Erlebnissen wahrheitsgetreu bearbeitet, Aarau 1888, 4, 24 u. 39-40; H. Lüthi: Erinnerungen an eine fünfjährige Dienstzeit, $100 \mathrm{u}$. 113.

H. Seiler: Fünf Jahre in der französischen Fremdenlegion, 48; P. Strupler: Fünf Jahre Fremdenlegion, 54, 225-226 u. 228; Fred Westphal (Hg.): Gemarterten-Schreie in die Kulturwelt. Briefe deutscher Söhne aus der Hölle der Fremdenlegion, Stuttgart 1931, 85-86; A[dolphus] R[ichard] Cooper: March or bust. Adventures in the Foreign Legion, London 1972, 14; Friedrich D.: Ananas-Granaten hatten wir mehr als Frühstück. Friedrich D., Legionär von 1953 bis 1955, in: D. Michelers: Le boudin, 67-82, hier 76.

F. Westphal: Gemarterten-Schreie, 85-86.

H. Seiler: Fünf Jahre in der französischen Fremdenlegion, 48; P. Strupler: Fünf Jahre Fremdenlegion, 152 u. 225-226. 
110 Christian Koller: Leiden und «Lust» in der Fremdenlegion

\section{Der Leiden zufügende Legionär}

Die Imperialkriege des 19. und 20. Jahrhunderts zeichneten sich durch eine besondere Brutalität und die Missachtung des europäischen Kriegs- und Völkerrechts sowie anderer ethischer und rechtlicher Normen aus. ${ }^{29}$ Dabei machten die im Namen der mission civilisatrice geführten Eroberungs-, «Pazifizierungs-» und AntiDekolonisationskriege Frankreichs, des Mutterlands der Menschenrechte, keine Ausnahme. ${ }^{30}$ Als Höhenkämme einer ganzen Eisbergkette aus dem 20. Jahrhundert bekannt sind etwa die Massaker von Sétif, Guelma und Kherrata vom Mai 1945, bei denen Kolonialfranzosen und Angehörige der französischen Streitkräfte Tausende algerischer Muslime töteten, ${ }^{31}$ die Massaker bei der Niederschlagung des madagassischen Aufstands von 1947/48, denen vermutlich Zehntausende zum Opfer fielen, ${ }^{32}$ die Auslöschung ganzer Dörfer und ihrer Bevölkerungen während des Indochinakriegs (wie etwa das Massaker von Mỹ Trạch vom 29. November 1947, bei dem über 300 Frauen, Kinder und alte Menschen ermordet wurden) oder die systematische Anwendung von Folter während der

Vgl. Dierk Walter: Organisierte Gewalt in der europäischen Expansion. Gestalt und Logik des Imperialkrieges, Hamburg 2014.

Vgl. z.B. Yves Benoit: Massacres coloniaux, 1944-1950. La IVe République et la mise au pas des colonies françaises, Paris 1995.

Vgl. Radouane Ainad-Tabet: Le 8 Mai 1945 en Algérie, Alger 1985; Charles-Robert Ageron: Mai 1945 en Algérie. Enjeu de mémoire et histoire, in: Matériaux pour l'histoire de notre temps 39 (1995) 52-56; Boucif Mekhaled: Chronique d'un massacre. 8 mai 1945, Sétif - Guelma - Kherrata, Paris 1995; Jean Louis Planche: Sétif 1945. Histoire d'un massacre annoncé, Paris 2006; Marcel Reggui: Les massacres de Guelma. Algérie, mai 1945. Une enquête inédite sur la furie des milices coloniales, Paris 2006; Guy Pervillé: Sétif. Enquête sur un massacre, in: L'Histoire 318 (2007) 44-49; Roger Vétillard: Sétif, mai 1945. Massacres en Algérie, Paris 2008; Mehana Amrani: Le 8 mai 1945 en Algérie. Les discours français sur les massacres de Sétif, Kherrata et Guelma, Paris 2010; Maurice Villard: Le 8 mai 1945. Sétif, Guelma, Le Constantinois, Montpellier 2010.

Vgl. Jacques Tronchon: L'Insurrection malgache de 1947, Paris 1986; Jean Eugène Duval: La révolte des sagaies - Madagascar 1947, Paris 2002; Jean-Luc Raharimanana: Madagascar 1947, Paris 2007. 
111 Christian Koller: Leiden und «Lust» in der Fremdenlegion

Dekolonisationskriege in Indochina und Algerien. ${ }^{33}$ Bereits bei den Eroberungen und «Pazifizierungen» von afrikanischen und asiatischen Territorien im 19. Jahrhundert hatten sich die französischen Armeen ähnlicher Praktiken bedient. ${ }^{34}$ An vielen dieser Aktionen waren Einheiten der Fremdenlegion beteiligt. Nach dem Zweiten Weltkrieg berichtete Albert Heyer der schweizerischen Militärjustiz über seine Erlebnisse während eines Aufstandes in Tunesien: «Dass die Legion sich wie eine Räuberbande aufführte, tötete, brandschatzte, plünderte und Frauen vergewaltigte, sei nur nebenbei bemerkt.» ${ }^{35}$

Über die Grausamkeiten gegen sogenannte «Wilde» berichteten Legionäre, die an der Eroberung Indochinas beteiligt waren, in ihren Memoiren freimütig und keineswegs immer anklagend oder

Vgl. Pierre Vidal Naquet: La Torture dans la République. Essai d'histoire et de politique contemporaine (1954-1962), Paris 1972; Rita Maran: Torture. The Role of Ideology in the French-Algerian War, New York, London 1989; Hamid Bousselham: Torturés par Le Pen, Alger 2000; Raphaëlle Branche, Sylvie Thénault: Le secret sur la torture pendant la guerre d'Algérie, in: Matériaux pour l'histoire de notre temps 58 (2000) 57-63; Hamid Bousselham: Quand la France torturait en Algérie, Alger 2001; Raphaëlle Branche: La torture et l'armée pendant la guerre d'Algérie, Paris 2001; Pierre Vidal Naquet: Les Crimes de l'armée française en Algérie 1954-1962, Paris 2001; Raphaëlle Branche: Des viols pendant la guerre d'Algérie, in: Vingtième Siècle 75 (2002) 123-132; Mohamed Harbi, Benjamin Stora (éd.): La Guerre d'Algérie, 1954-2004. La fin de l'amnésie, Paris 2004; Damien Carron: Les dossiers de jugement des légionnaires suisses. Source inédite pour une histoire de la torture pendant la guerre d'Algérie, in: Mauro Cerutti et al. (éd.): Penser l'archive, Lausanne 2006, 303-314; Marnia Lazreg: Torture and the Twilight of Empire, Princeton 2007; Fabian Klose: Menschenrechte im Schatten kolonialer Gewalt. Die Dekolonisierungskriege in Kenia und Algerien 1945-1962, München 2009.

34 Vgl. z.B. zur Eroberung Algeriens: Lucien-François de Montagnac: Lettres d'un soldat. Neuf Années de campagnes en Afrique. Correspondance inédite du colonel de Montagnac, Paris 1885, passim; Olivier Le Cour Grandmaison: Coloniser, Exterminer. Sur la guerre et l'État colonial, Paris 2005.

35 Schweizerisches Bundesarchiv (Bern) E 5330-1/1975/95/1952/335 Militärgericht: Ik. Kan. Heyer, Albert, 1952. 
schuldbewusst. ${ }^{36}$ So schilderte der Schweizer Robert Kaufmann eine Aktion mit lapidaren Worten: «Am 8. Dezember 1885 umringten wir die Feinde in einem Dorfe an der chinesischen Grenze, zündeten dasselbe an und bewachten die Ausgänge. Sämmtliche Tonkinesen starben den Feuertod.» ${ }^{37}$ Der Deutsche Fred Westphal wirkte in der Zwischenkriegszeit in Syrien an Expeditionen gegen Dörfer mit, die im Verdacht standen, mit Rebellen zu kollaborieren, und rechtfertigte die dabei als Kollektivbestrafung vorgenommenen Zerstörungen und Massaker folgendermassen:

In den arabischen Dörfern [...] vergalten wir den Banditen ihre Schandtaten. Die Männer wurden zusammengetrieben und zum abschreckenden Beispiel an den Obstbäumen aufgehängt, das Dorf danach verbrannt. Als Kulturmensch muss man dieses Vorgehen verurteilen, aber man muss bedenken, dass ohne diese Vergeltung die ganze christliche Bevölkerung des Libanon von den entmenschten und fanatischen Banditen ermordet worden wäre. [...] Durch die arabischen Dörfer ging ein panischer Schrecken. «Rettet euch, die Legion kommt! » ${ }^{38}$

Auch in den Dekolonisationskriegen nach 1945 waren ähnliche Aktionen gegen die Zivilbevölkerung keine Seltenheit. Mehrere Ex-Legionäre erinnerten sich aus dem Indochinakrieg an die sogenannte carte blanche, bei der der Kollaboration mit den Viêt Minh verdächtige Dörfer zerstört und ihre Bewohnerinnen und Bewohner kollektiv bestraft wurden. Friedrich D. erinnerte sich an die folgende Episode, die auf die Ermordung einiger Legionäre folgte:

Wir mit den LKW's durchs Dorf gefegt, gebrüllt: «Alle Männer, die älter als fünfzehn Jahre sind, raus!» - Haben sie hinter die Hütten geführt und gefragt: «Wer war's?» - Keine Antwort. Wurde das Maschinengewehr aufgebaut, noch mal gefragt. - Wieder keine Antwort. - Abgemäht. Alle Mann. Danach den ganzen Scheiss angesteckt. Konnten Sie die Frauen laufen sehen! Mit Sack und Pack aus den Hütten. Die brannten doch wie Zunder. ${ }^{39}$

Vgl. z.B. W. Bolliger: Aus dem Leben und Treiben der Fremdenlegion, 33-34; R. Kaufmann: Feldzug in Afrika, 21 u. 39-40; Jean Trüb: Erlebnisse eines Fremdenlegionärs in Algerien und Tonking, Horgen [1887?], 56. R. Kaufmann: Feldzug in Afrika, 39.

38 F. Westphal: Gemarterten-Schreie, 142-143.

39 F. D., Ananas-Granaten, 76. 
113 Christian Koller: Leiden und «Lust» in der Fremdenlegion

Gewisse mündliche Zeugnisse liessen selbst im zeitlichen Abstand von mehreren Jahrzehnten immer noch einen Nachhall der euphorischen Stimmung erkennen, die sich bei solchen Aktionen offenbar entfaltete. Hans-Friedrich G. berichtete, carte blanche sei «für uns das Grösste» gewesen:

Alles niedermachen. Selbstverständlich zündeten wir zunächst die cannejas an. Das Schilf brannte wie Zunder. Dann knallten wir alles nieder, was uns vor die Füsse kam. [...] Das durften wir. Verbrannte Erde, ganz klar. Aber wir durften das, ohne bestraft zu werden. Wir sahen das nicht so eng und unsere Offiziere ebenso wenig. Die machten es uns sogar vor. ${ }^{40}$

Die in diesen Kriegen weit verbreiteten Folterungen von Gefangenen wurden in den Memoiren mancher Ex-Legionäre verurteilt. ${ }^{41}$ Michael Donovan wies aber auch auf die Faszination hin, mit der er und seine Kameraden in den 1920er Jahren der öffentlichen Tortur eines algerischen Rebellen beiwohnten:

Nach dem Essen versammelten wir uns im Kreis um die Gefangenen, schwatzten, spekulierten und stupsten uns gegenseitig wie Schuljungen, die auf den Beginn einer Filmvorführung warteten. Wir sahen alle erwartungsvoll einer Form von Unterhaltung entgegen, da wir wussten, dass die Gefangenen bald einem Verhör nach Legionsart unterzogen würden. Der Offizier befahl, einen der Gefangenen an einen Pfahl in der Nähe seines Zeltes zu binden, und nachdem dies getan war, stellte er ihm einige Fragen, zuerst auf Arabisch und dann auf Französisch. Der Gefangene antwortete nicht [...]. Aus der Glut des Kochfeuers wurde ein Feuer entfacht und in dieses wurde einer der Eisenstäbe gehalten, die wir für die Reinigung der Maschinengewehrläufe benutzten. Als der Stab glühend heiss war, wurde er aus dem Feuer gezogen und gegen die Fusssohlen des Arabers gepresst. Ein schwacher Rauch stieg auf, und ich roch anbrennendes Fleisch, was mich anwiderte und dennoch faszinierte. Hier war ich, etwas mehr als einen Monat weg von zu Hause, und wohnte fasziniert der Folterung eines Mitmenschen mit einer glühenden Stange bei. ${ }^{42}$

Hans-Friedrich G.: Ich wollte nur raus aus dem kaputten Deutschland. Hans-Friedrich G., Legionär von 1952 bis 1957, in: D. Michelers: Le boudin, 55-66, hier 61.

H. Ainley: In order to die, 30; Horst A.: Ich hab immer gesagt: Männer bleibt Mensch. Horst A., Legionär von 1953 bis 1957, in: D. Michelers: Le boudin, 83-100, hier 90-91 u. 97.

Michael Donovan: March or Die! London 1932, 102: «When soupe was past, we all gathered in a circle round the prisoners, talking, speculating 
114 Christian Koller: Leiden und «Lust» in der Fremdenlegion

Inwiefern es sich bei diesen Gewaltexzessen, denen eine komplexe Kombination struktureller und situativer Faktoren zugrunde lag, auch um Akte von Sublimation handelte, müsste eine psychoanalytisch angelegte Untersuchung klären. Auf andere Praktiken zur Befriedigung und Stillung der «Lust» des Legionärs wird im folgenden Abschnitt eingegangen.

\section{Die «Lust» des Legionärs}

Der eingangs erwähnte Anthony Delmayne hielt 1958 in seinen Memoiren fest, die Legionäre würden Sexualität eher als lästigen Trieb denn als erotisches Vergnügen betrachten:

Unsere Einstellung zum Sex war nicht gerade positiv oder kreativ. Wir betrachteten Lust und Begehren als ein Ärgernis, das zu beseitigen war, und vollzogen den Geschlechtsakt, ob mit einem Mann oder einer Frau, mehr zur Erleichterung als zum Vergnügen. ${ }^{43}$

Der Hinweis auf die beinahe beliebige Lustbefriedigung mit Männern oder Frauen ist bemerkenswert und verdient eine

and nudging one another like a lot of schoolboys awaiting the start of some film show. All of us fully expected an entertainment of some kind, for we knew that the prisoners would soon be put to the Legion's form of questioning. The officer ordered that one of the prisoners be tied to a pole stuck in the sand near his tent, and when this was done put a number of questions to him, first in Arabic and then in French. The prisoner made no attempt to answer [...]. A fire was kindled from the embers of the cook's fire, and into this was placed one of the iron rods which we used for the cleaning of machine-gun barrels. When the rod was redhot, it was withdrawn from the fire and pressed against the soles of the Arab's feet. A faint puff of smoke went up and I smelt burning flesh, which sickened me yet fascinated me. Here was I, little more than a month away from home, sitting fascinated at the sight of a fellow-man being tortured with a red-hot bar.» (eigene Übersetzung).

43 A. Delmayne: Sahara Desert Escape, 100: «Our attitude to sex was not exactly positive or creative. We regarded desire as a nuisance that had to be removed, and whether with a man or a woman we went into the sexual act more for relief than for pleasure» (eigene Übersetzung). 
115 Christian Koller: Leiden und «Lust» in der Fremdenlegion

genauere Analyse. ${ }^{44}$ Homosexuelle Beziehungen tauchten in Selbstzeugnissen immer wieder auf und wurden gelegentlich als «Legionslaster ${ }^{45}$ bezeichnet. Auch die Fremdenlegionsforschung hat das Thema wiederholt aufgegriffen, es jedoch selten einer vertieften Analyse unterzogen. Die Forschung zu Homosexualität und Kolonialismus hat erst in den letzten Jahren an Fahrt gewonnen, insbesondere mit den Arbeiten von Robert Aldrich. ${ }^{46}$ Dieser begründet Homosexualität in den Kolonialarmeen mit einem homosozialen Ethos, einer homoerotischen Ästhetik (Jugend- und Vitalitätskult, Uniformfetischismus), der zentralen Bedeutung kameradschaftlicher Solidarität und schliesslich der Abwesenheit von Frauen. ${ }^{47}$

Da Homosexualität während der behandelten Periode negativ konnotiert war, ist eine Analyse der Selbstzeugnisse in dieser

Vgl. für das Folgende auch Christian Koller: Sex as a Transcultural Event? Sexualities in the French Foreign Legion and their Representations in Autobiographical Writing, in: Sebastian Jobs, Gesa Mackenthun (eds.): Embodiments of Cultural Encounters, Münster 2011, 75-92; André-Paul Comor: Les plaisirs des légionnaires au temps des colonies. L'alcool et les femmes, in: Guerres mondiales et conflits contemporains 222 (2006) 33-42.

Zum Beispiel Erwin Rosen [Pseud. für Erwin Carlé]: In der Fremdenlegion. Erinnerungen und Eindrücke, Stuttgart ${ }^{81909, ~ 277 ; ~ P . ~ S t r u p l e r: ~}$ Fünf Jahre Fremdenlegion, 37.

Robert Aldrich: Weisse und farbige Männer. Reisen, Kolonialismus und Homosexualität, in: Forum: Homosexualität und Literatur 7 (1989) 5-24; ders.: Homosexuality in the French colonies, in: Jeffrey Merrick, Michael Sibalis (eds.): Homosexuality in French History and Culture, New York 2001, 201-218; ders.: Colonialism and Homosexuality, London 2003. Ausserdem: Joseph A. Boone: Vacation cruises; or, the homoerotics of Orientalism, in: Publications of the Modern Language Association 110 (1985) 89-107; Rudi Bleys: Homosexual Exile. The textuality of the imaginary paradise, 1800-1980, in: Journal of Homosexuality 25 (1993) 165182; ders.: The Geography of Perversion. Male-to-Male Sexual Behaviour outside the West and the Ethnographic Imagination, 1750-1918, New York 1995; Christopher Lane: British Colonial Allegory and the Paradox of Homosexual Desire, Durham 1995; Lee Wallace: Outside History. Same-Sex Sexuality and the Colonial Archive, in: S. Jobs, G. Mackenthun: Embodiments of Cultural Encounters, 61-74. R. Aldrich: Colonialism and Homosexuality, 79-80. 
Hinsicht nur beschränkt ergiebig. Kein einziger Autor berichtet von eigenen Erfahrungen mit gleichgeschlechtlicher Sexualität. Homosexualität wurde in aller Regel nur im Kontext genereller Kritik an der Fremdenlegion thematisiert; Autoren, die ein ausgewogenes oder gar positives Bild von der Söldnertruppe zu vermitteln suchten, sprachen nicht darüber. Eine Ausnahme bildet Friedrich Glauser, der das Thema in seinem autobiographischen Legionsroman an verschiedenen Stellen ohne homophobe und anklagende Untertöne einflocht - was ihm übrigens lange Diskussionen mit seinem skeptischen Verleger bescherte. ${ }^{48}$

Trotzdem lassen sich aus den Quellen einige Grundtendenzen ausmachen. Die meisten Schreiber, die sich Gedanken über die Ursachen gleichgeschlechtlicher Sexualpraktiken machten, gingen zumindest implizit von einem Konzept situativer Homosexualität aus. Für den in der Zwischenkriegszeit schreibenden deutschen Ex-Legionär Franz Glienke war dieses Konzept plausibel:

Die Truppen liegen manchmal jahrelang in Einsamkeit und Wüste. Die Frauen, die zu sehen sind, sind derart verseucht, dass schon der blosse Anblick ein Grauen erweckt. Geld ist auch meist die schwache Seite. Was Wunder, dass sich die Erotik andere Bahnen sucht, um zu ihrem Recht zu kommen. ${ }^{49}$

In zahlreichen Selbstzeugnissen finden sich Belege dafür, dass es bei der Homoerotik in der Fremdenlegion nicht nur, manchmal sogar überhaupt nicht um simple sexuelle Triebabfuhr ging. Verschiedene Memoiren und Briefe thematisierten gleichgeschlechtliche Zweierbeziehungen, die eine starke emotionale Komponente beinhalteten und von Stabilität gekennzeichnet waren. Verschiedentlich ist in den Quellen von «Paaren» oder gar «Ehepaaren» die Rede. Der Österreicher Raimund Anton Premschitz empörte sich kurz nach der Wende zum 20. Jahrhundert über die

traurige Ursache von so vielen Unzuchtsfällen, welche hier vorkommen und eigentümlicherweise stillschweigend geduldet werden. Dieselben

48 Vgl. F. Glauser: Gourrama, 317.

49 Franz Glienke: Ein Prolet in der Fremdenlegion, Berlin, Wien 1931, 37.

Vgl. z.B. auch F. Westphal: Gemarterten-Schreie, 151. 
117 Christian Koller: Leiden und «Lust» in der Fremdenlegion

werden ganz öffentlich durchgeführt ohne jedwedes Schamgefühl, als wäre dies eine selbstverständliche Sache. Täglich sieht man solche Paare wie Mann und Frau zusammenleben. ${ }^{50}$

In der Zwischenkriegszeit thematisierte Glauser in Gourrama verschiedene gleichgeschlechtliche Verhältnisse. So pflegten im Roman die Legionäre Schilasky und Todd eine liebevolle Beziehung. Während Todd vor seinem Eintritt in die Legion offenbar keine homosexuellen Erfahrungen hatte, fühlte sich Schilasky seit seiner Jugend zu Männern hingezogen und litt immer noch unter dieser Neigung. ${ }^{51}$ Die Legionsbehörden tolerierten homoerotische Beziehungen, so lange sie nicht zu stabil wurden und die Loyalität zur Institution zu untergraben drohten.

Ein weiterer Aspekt der Sexualität in der Fremdenlegion war die in den dominanten europäischen Moraldiskursen negativ bewertete Prostitution..$^{52}$ War käuflicher Sex in den vormodernen Söldnerheeren, in denen es sogar die militärische Funktion des «Hurenweibels» gab, nicht besonders brisant gewesen, so wurde das Thema mit dem Übergang zu den nationalstaatlichen Bürgerarmeen tabuisiert, ohne in der Realität zu verschwinden. Die französische Armee richtete in den Kolonien bereits vor dem Ersten Weltkrieg Truppenbordelle ein. ${ }^{53}$ Dahinter stand die Furcht vor der Infizierung der Soldaten mit Geschlechtskrankheiten durch unkontrollierte Prostitution. Diese zu Ende des Ersten Weltkrieges als BMC institutionalisierten Einrichtungen - die Abkürzung konnte wahlweise für Bordel Militaire Contrôlé oder Bordel Mobile de Campagne stehen -, ${ }^{54}$

50 R. A. Premschitz: Meine Erlebnisse als Fremdenlegionär, 116.

51 F. Glauser: Gourrama, 122.

52 Vgl. auch J. Laffin: The French Foreign Legion, 38-44; D. Porch: The French foreign legion, 309-310 u. 387; A.-P. Comor: Les plaisirs des légionnaires, 39-42.

53 Vgl. J. Laffin: The French Foreign Legion, 38; Jean-Yves Le Naour: Régénération ou dépravation? Moralisation, angoisse sexuelle et anomie dans la France de la Première Guerre mondiale, Diss. phil. (masch.), Université de Picardie 2000, I 306.

54 Vgl. Michel-Serge Hardy: BMC et prévention sanitaire, in: Revue historique des armées 194 (1994) 38-43; ders.: De la morale au moral des troupes ou l'histoire des BMC (1918-2004), Paris 2004. 
118 Christian Koller: Leiden und «Lust» in der Fremdenlegion

existierten in der Folge an allen Legionsstandorten und wurden häufig auch bei Feldzügen und Manövern mitgeführt. ${ }^{55}$ Ähnlich wie die Fremdenlegionäre waren die Sexworkerinnen mit Zeitverträgen angestellt. ${ }^{56}$ Da das 1946 erlassene Verbot von Bordellen in Frankreich sich nur auf das Mutterland bezog, florierte in den Kolonien die Prostitution im Umfeld der Fremdenlegion und anderer französischer Institutionen weiter. Erst 1974 wurden die BMC offiziell abgeschafft. ${ }^{57}$

Neben den BMC gab es in Nordafrika Bordellstädte, so in Sidibel-Abbès und im nordmarokkanischen Meknès. Die rund 500 Gebäude umfassende Bordellstadt von Meknès, die bis zur marokkanischen Unabhängigkeit von 1956 in Betrieb war und auch Cafés, Restaurants, Tanzlokale und medizinische Versorgungsstellen umfasste, war in zwei Sektoren aufgeteilt. An Tagen mit geraden Daten durften Fremdenlegionäre den Nordteil und einheimische Männer den Südteil besuchen, an Tagen mit ungeraden Daten war es umgekehrt. Auch in Südostasien blühte im Umfeld der französischen Kolonialmacht und ihrer Soldaten die kontrollierte und unkontrollierte Prostitution. In Saigon existierten zur Zeit des Indochinakrieges mehrere Grossbordelle, so der Parc aux Buffles mit etwa 400 und das - nach einem französischen Marschall benannte - Gallieni mit etwa 300 Sexworkerinnen. ${ }^{58}$

Vgl. z.B. H. A.: Ich hab immer gesagt, 86; Marc O. Eberle: In fremden Diensten - Deutsche Legionäre im Indochinakrieg (ARTE 2004), min. 10:01ff. Vgl. z.B. C. John: Nothing to Lose, 151; Mustapha El Quadéry: Femmes des Bordels Militaires de Campagne. Les BMC de l'armée coloniale française au Maroc, in: M. Cheikh, M. Péraldi (éd.): Des femmes sur les routes. Voyage au féminin entre Afrique et Méditerranée, Casablanca 2009, 229-244.

Vgl. Marie-Victoire Louis: Interview de Mademoiselle de Liancourt. A propos de la prostitution en période de guerre, suivi d'annexes (3.1.1991) www.marievictoirelouis.net/document.php?id=493\&themeid (27.6.2011). Allgemein zur Kolonialprostitution in Nordafrika: Christelle Taraud: Le prostitution coloniale. Algérie, Tunisie, Maroc (1830-1962), Paris 2003. Vgl. z.B. C. John: Nothing to Lose, 190; Hans E. Bauer: Verkaufte Jahre. Ein deutscher Fremdenlegionär berichtet über seine Erlebnisse in Indochina und Nordafrika, Gütersloh 1957, 235; H.-F. G.: Ich wollte nur raus, 
119 Christian Koller: Leiden und «Lust» in der Fremdenlegion

Die Prostitution im Umfeld der Fremdenlegion reflektierte einerseits besonders drastisch die Überkreuzung und gegenseitige Verstärkung von kolonialem Machtgefälle und asymmetrischer Geschlechterordnung. Sie stellte den kolonisierten weiblichen Körper zur einigermassen beliebigen Disposition der Kolonisatoren und verletzte die sexualmoralischen Vorstellungen, die religiösen Normen und den Ehrenkodex der kolonisierten Gesellschaften in gravierender Weise. Auf der anderen Seite war das Bordell, wie verschiedene Legionärsselbstzeugnisse zeigen, auch ein Ort vielfältiger und durchaus reziproker Akkulturationsprozesse. Erschienen die Angehörigen kolonisierter Gesellschaften in den meisten Memoiren primär als stereotypisierte Exoten, so erhielten die Sexworkerinnen zuweilen ein individuelles Gesicht und manchmal sogar einen Namen. Die Prostitution scheint also zum Abbau rassistischer und exotistischer Stereotypen beigetragen zu haben, wobei dieser Effekt aber in den meisten Fällen nur kurzlebiger Natur gewesen sein dürfte. Darüber hinaus konnte sich das Verhältnis zwischen Legionären und Sexworkerinnen, die letztlich in einem ähnlichen, ihren Körper temporär zur Disposition stellenden Vertragsverhältnis zur Kolonialmacht standen, manchmal in einem kameradschaftlichen Sinne gestalten. Im Weiteren boten die Legionsbordelle einzelnen einheimischen Frauen auch rare Karrierechancen im Umfeld des Kolonialstaates, wurden die BMC doch in der Regel von einer (häufig nordafrikanischen) Madame geleitet. ${ }^{59}$

Daneben gab es auch dauerhaftere Beziehungen zwischen Legionären und einheimischen Frauen. Bis zum Ersten Weltkrieg schwiegen sich die meisten schreibenden Ex-Legionäre zur dieser offenbar heiklen Thematik aus oder äusserten sich nur verklausuliert. ${ }^{60} \mathrm{Ab}$ der Zwischenkriegszeit wurden einige Autoren offener

60; Michel Bodin: Le plaisir du soldat en Indochine (1945-1954), in: Guerres Mondiales et Conflits Contemporains 222 (2006) 7-18.

59 Vgl. H. Ainley: In order to die, 59; C. John: Nothing to Lose, 233; S. Murray: Legionnaire, 46; Philip Rosenthal: Einmal Legionär, Hamburg 1980, 61-63 u. 78-81; F. Glauser: Gourrama, 157-170.

60 Vgl. z.B. Emil Wälti: Fieberschub und Saufgelage. Als Fremdenlegionär in Schwarzafrika 1894/95, hg. von Paul Hugger, Zürich 1999, 69. 
und erwähnten kürzere und längere Beziehungen zu lokalen Frauen in Nordafrika, Syrien und Indochina. In Glausers Gourrama unterhalten sowohl der dem Autor stark nachempfundene Korporal Lös als auch Leutnant Lartigue Verhältnisse zu Marokkanerinnen. Die Liaison zwischen Lös und der Marokkanerin Zeno wird dabei durch Geldgeschenke an Zenos Familie eingefädelt. ${ }^{61}$ Ein ähnliches Phänomen war in Indochina weit verbreitet: die Ehen auf Zeit zwischen Legionären und sogenannten Congaï. ${ }^{62}$ Für die Legionäre boten diese Beziehungen die Möglichkeit einer relativ stabilen Partnerschaft, die über die Sexualität hinaus eine emotionale Komponente beinhaltete und sie zudem von ungeliebten «weiblichen» Aufgaben des Garnisonsdienstes entlastete, aber dennoch keine unbefristeten Verpflichtungen nach sich zog.

Solche Beziehungen von Fremdenlegionären zu afrikanischen und asiatischen Frauen ausserhalb der offenen Prostitution erscheinen als ein kulturell und sozial äusserst komplexes Phänomen. Die Praxis des Kaufs und Verkaufs von «Ehe»-Frauen erscheint als Mischung aus traditionellen lokalen Gebräuchen (Brautpreis) und Menschenhandel beziehungsweise offener Prostitution. Sie wurde angetrieben vom spezifischen Bedürfnis der Söldner nach einer stabileren und emotional befriedigenderen Alternative zum Bordellbesuch. Die Akkulturationsprozesse im Kontext dieser Verhältnisse waren mannigfaltig. Erstens kamen Afrikanerinnen und Asiatinnen in engen Kontakt zu einzelnen Europäern und eigneten sich europäische Praktiken wie den Gebrauch der französischen Sprache und der französischen Währung und die Wertschätzung europäischer Kleidermode an. Zweitens wurden die Legionäre ihrerseits in einer gewissen Weise Teil asiatischer oder afrikanischer Familien, was, wie sich aus autobiographischen Berichten zur Thematik schliessen

$61 \quad$ F. Glauser: Gourrama, 57-58.

62 J. Laffin: The French Foreign Legion, 43-44 u. 132-133; Ann Laura Stoler: Making Empire Respectable. The Politics of Race and Sexual Morality in Twentieth-Century Colonial Cultures, in: American Ethnologist 16 (1989) 634-660; D. Porch: The French foreign legion, 221-222 u. 507-508; M. O. Eberle: In fremden Diensten, min. 11:06ff. 
lässt, zumindest in einem beschränkten Rahmen zum Abbau rassistischer und exotistischer Stereotypen beigetragen haben mag. Drittens entstand mit den diesen Verhältnissen entsprungenen Kindern eine Gruppe von Menschen in-between, die zur Rolle als kulturelle Broker zwischen Kolonisatoren und Kolonisierten geradezu prädestiniert waren. Diese Akkulturationsprozesse fanden indessen stets im Kontext intersektionaler Asymmetrien statt, indem sich in den Beziehungen zwischen Legionären und einheimischen Frauen das grundsätzliche koloniale Machtgefälle, die asymmetrischen Geschlechterordnungen sowohl der europäischen wie der kolonisierten Gesellschaften sowie ökonomische Ungleichheiten potenzierten. Hinzu kam die Brüskierung einheimischer Männer, die sich nicht nur in ihrer maskulinen Ehre verletzt, sondern auch in ihren Chancen auf dem Heiratsmarkt beschnitten sahen und entsprechenden Groll gegen Fremdenlegionäre und andere Europäer hegten.

Mutatis mutandis lebten solche Praktiken in der Fremdenlegion auch nach dem Ende der französischen Kolonialherrlichkeit weiter. In den 1980er Jahren hat die Soziologin Rosemary McKechnie in einer ethnographischen Studie über Frauen im Umfeld des Legionsstützpunktes Calvi auf Korsika ein breites Feld zwischen Prostituierten und Offiziersehefrauen festgestellt. Zahlreicher als die Prostituierten war die vor allem aus Nordafrikanerinnen mit geringer Bildung bestehende «floating population» von Frauen, die in gelegentlicher Weise von den Legionären lebten. Viele von ihnen arbeiteten in Bars oder hielten sich dort die grösste Zeit auf. Manchmal schliefen sie gratis mit den Männern, manchmal verlangten sie «Geschenke», und oft zahlten die Verehrer für ihre Kleider oder Wohnungen. In der Regel, so McKechnie, gelinge es diesen sowohl in der Legion als auch in der umliegenden Zivilgesellschaft stigmatisierten Frauen recht schnell, sich mit einem Legionär auf einer längerfristigen Basis zu verbinden. ${ }^{63}$

63 Rosemary McKechnie: Living with images of a fighting elite. Women and the Foreign Legion, in: Sharon Macdonald et al. (eds.): Images of Women in Peace and War. Cross-Cultural and Historical Perspectives, London 1987, 122-147, hier 135. 
Insgesamt erscheint die französische Fremdenlegion als ein Mikrokosmos, der die Analyse des Begriffspaares Lust und Leiden aus historischer, kulturwissenschaftlicher und sozialpsychologischer Perspektive unter ganz spezifischen Rahmenbedingungen gestattet, nämlich im Kontext einer männerbündischen und straff hierarchischen Struktur, die sich überwiegend in kolonisierten Gebieten bewegte und zugleich Gegenstand umfangreicher medialer und kultureller Projektionen war. Insofern wäre eine Verallgemeinerung der hier ausgebreiteten Befunde zu anthropologischen Grundkonstanten sicherlich problematisch, verschiedene der angesprochenen Phänomene erscheinen aber gleichsam als Radikalisierung allgemeinmenschlicher Probleme.

conexus $1(2018) 97-122$

(C) 2018 Christian Koller. Dieser Beitrag darf im Rahmen der Lizenz CC BY-NC-ND 4.0 - Creative Commons: Namensnennung/nicht kommerziell/keine Bearbeitungen - weiterverbreitet werden.

(C) $\odot \otimes \Theta$

https: / / doi.org/10.24445/ conexus.2018.01.008

Prof. Dr. Christian Koller, Schweizerisches Sozialarchiv, Stadelhoferstrasse 12, 8001 Zürich. - Universität Zürich, Historisches Seminar, Karl Schmid-Strasse 4, 8006 Zürich

koller@sozarch.uzh.ch chkoller@hist.uzh.ch 